The BMJ

mlooi@bmj.com

Cite this as: $B M J 2020 ; 369: \mathrm{m} 2221$ http://dx.doi.org/10.1136/bmj.m2221 Published: 5 June 2020

\section{Risky Business: lessons from covid-19}

\section{Clinical professionals are at the forefront of learning and innovation about, and sparked by, the pandemic. Mun-Keat Looi and Rebecca Coombes report from the first virtual Risky Business conference, held on 2 June}

Mun-Keat Looi, Rebecca Coombes

Video 1 The science clinical trials: what we know and the pipeline

"We need to remember what we can do, not what we can't."

The opening words from Matthew Shaw, chief executive of Great Ormond Street Hospital, were in relation to the Nightingale hospitals-and captured the mood of the conference. Five months since the UK's first confirmed case of covid-19 and three since its lockdown began, the world is a different place. But among the chaos, exhaustion, and grief, there is much we have learnt and achieved.

\section{No magic bullet}

"It's unlikely there's one drug that will suddenly knock out coronavirus and everything will be back to normal," Martin Landray, co-chief collaborator of the RECOVERY trial, the world's largest clinical trial of treatments for covid-19, said. " ${ }^{1}$ But even moderate effects, such as reducing the number of hospital patients dying by just a fifth, is a colossal improvement."

Video 2 The people: the science of wellbeing

The RECOVERY trial is a platform study, meaning it is considering several treatments within one trial. Starting in mid-March, there were just nine days between the first drafting of the protocol and recruitment of the first patients, including regulation and ethics provisions.

There are no drugs for covid-19 that have been shown to work, with the possible exception of remdesivir, Landray, a professor of medicine and epidemiology at the University of Oxford, pointed out. "The early data for remdesivir looks encouraging for reducing the time for hospital patients to improve and go home, but we still don't have any evidence that it reduces mortality," he said.

In the UK the mortality of patients admitted to hospital is extremely high, at $20-25 \%$. "One in every four to five patients admitted to hospital will not survive that admission and we need to focus, in the first instance, on treatments that will improve that," he added.

If any one, or a combination, of the drugs under trial shows promise in reducing deaths, the gains will be significant. "In the US, there were roughly 25000 deaths per week over the past few months. If you could reduce that by a quarter it would mean 5000 lives saved every week," Landray said.

Video 3 The medicine ICU panel discussion: what have we learnt?

\section{Poor surveillance equals risk}

The scientific models used to predict how different situations might play out are only as good as the data available to inform them.

"We've been doing some work on estimating how much underreporting there was at the beginning of the pandemic," Adam Kucharski, a lecturer in mathematical modelling at the London School of Hygiene and Tropical Medicine, said. "If we look back at that period in mid-March, in a lot of European countries fewer than $5 \%$ of people who had symptoms were showing up in the data as cases. So, we're seeing a tiny fraction of what was going on.

"This is important because as we lift lockdown measures, if we don't have good surveillance to identify infections we're only going to notice a flare up when a lot of cases start appearing in intensive care. That's something to be cautious about: globally, areas that have poor surveillance take much longer to spot that they have made a mistake."

In common with other European countries, the UK still has a high level of infection, he said, and we could expect to see this prevalence plateau for a long period of time. "A lot of countries across Europe that are lifting lockdown measures still have hundreds, if not thousands, of infections a day and even if the reproduction number doesn't go above one, cases will remain flat. That, however, is still thousands of infections and the health burden that goes along with that."

\section{Immense mobilisation effort}

"We initially thought covid-19 was a lung disease," Nick Hart, joint clinical director at Guy's and St Thomas' NHS Foundation Trust, said, "but the patients came in sicker than we'd been led to believe." He emphasised that covid-19 is not a single disease-it's a complex multisystem inflammatory disease that attacks the brain, heart, lungs, and kidneys, and there is still much we don't yet know.

While the Nightingale hospitals grabbed the headlines, it's the practical innovations and personnel organisations that have kept the NHS functioning during the pandemic, he said. "It's not just where the beds go, but have we got enough oxygen, enough electricity?"

"There was an immense mobilisation of staff," he said. "By the time we reached peak we had 476 nurses deployed to intensive care. We had 243 doctors-consultants and junior doctors-trained to work alongside the intensive care team. You can say 
all that in about two minutes, but the actual amount of management that goes into expanding your bed base to that size is phenomenal."

Jim Down, consultant in anaesthesia and critical care at University College London Hospital, applauded the entirely new systems of working thrown together at speed to cope with the patient surge. These were based around teams-functional teams organised by task, for example to turn patients over or put in lines-drawn from all over his hospital. Learning, he said, has had to be rapid.

Rebecca Smith, senior sister in critical care at the Royal London Hospital, said that nurses have had to become "much more task oriented" to cope with the workload. New coordinator roles to keep track of referrals and bed availability were developed, freeing up other nurses for valuable bedside time with patients.

Covid-19 "is not a straightforward thing to look after," Down said. For example, nursing ratios based on initial models soon turned out to be inappropriate and a huge risk for any second wave. "I don't think we can safely manage these patients at 1:6 (nurse to patient) ratios," he said, acknowledging there are not the resources to go back to the usual intensive care standard of 1:1.

Down spoke of compassion in the face of protocol and the "balance between risk and humanity." All families were given full personal protective equipment (PPE) and allowed to say goodbye to loved ones who were dying. In a time of shielding, isolation, and remote triage, "it's important to maintain some face-to-face time," said Down, as so many people are dying.

Such experiences take their toll. Though the medical response has been phenomenal, "staff are exhausted" and need support, Hart said.

\section{Primary care forever changed}

It was fair to say that the primary care model had not changed in decades, Martin Marshall, chair of the Royal College of General Practitioners council, said. But it was propelled forward "dramatically" in just a few weeks in April. And, he said, such changes are more likely to stay the longer the pandemic continues and they form a new normal.

Marshall highlighted three ways in which general practice has changed forever: technology, administration, and public health. Before the pandemic a small number of practices used remote triage to direct patients to the most appropriate services-now $100 \%$ of practices do so. Before covid-19, 70-80\% of consultations were face-to-face-that's now just $15-20 \%$. There has been a massive increase in remote diagnostics and monitoring as well. Marshall did, however, point out that the systems most in use are still the "old tech" of phone calls and email.

He said there has been a 30\% reduction in "bureaucracy" such as contracts and appraisals, to the relief of many in general practice. This has restored a system of "high trust, less checking" that he hopes will remain after covid-19 passes.

That trust is crucial in the role of GPs in the public health effort. GPs are a first contact for patients and act as a link between them and the public health network, thus supporting infection control. That must be maintained, Marshall said, while recognising that it may take away from standard care duties that only primary care can fulfil. We don't want to risk diluting what it is that GPs do best, including care for longer term "diseases that don't go away," such as cancer and childhood conditions.

\section{Litigation}

Covid-19 has changed the doctor-patient relationship forever, Pallavi Bradshaw, medicolegal lead for risk prevention at the Medical Protection Society, said, and while the crisis may have "escalated the breakup between doctors and patients there may be a new love affair between society and the profession."

Patients have been physically and emotionally separated from doctors by PPE, remote consulting, and fear of accessing healthcare at all, Bradshaw said, which has strained the trust and communication on which the doctor-patient relationship is built.

Only 1-5\% of patients litigate after negligent treatment, but poor communication is often a differentiating factor between those who are sued and those who are not. This is a risk when telemedicine is impaired by the loss of verbal and non-verbal cues, and impatience. Bradshaw cited one study that showed doctors on remote consultations interrupted patients after 12-18 seconds when completion of the story would have taken 90 seconds.

Bradshaw questioned if the current respect and empathy shown by the press and public towards medical workers would translate into an increased tolerance of medical error. Society may understand that stretched staff and resources will not always deliver quality care and, on occasion, patients will be harmed. Despite the goodwill, there are fears that a "tsunami of claims" is approaching, owing to a disruption in care and aggravated by economic hardship, she said. With outstanding NHS liabilities estimated at over €8obn (€89bn; $\$ 100 b n)$, there is a looming worry over patients seeking compensation at this time.

\section{Inequality amplified}

On the day that the government released the Fenton report ${ }^{2}$ on disparities in risk and outcomes of covid-19, Yvonne Coghill, director of NHS Workforce Race Equality Standard, gave the conference a stark account of the situation: a black man is 4.2 times more likely to die of covid-19 than a white man (a black woman is 4.3 times more likely); the first 10 healthcare workers to die of covid-19 were all from black and minority ethnic (BAME) backgrounds; and by mid-May, of the 222 healthcare workers who had died of covid-19, $61 \%$ were BAME.

Make no mistake, Coghill said, this is about race inequality. Matters of wealth, education, judiciary, housing, and health are all things that people from BAME backgrounds cope with every day and such "microaggressions"-the consequences of "living in a society that is not built for you" -take a toll on psychological and physical health.

Coghill said the NHS needs a clear strategy for improvement for BAME staff working in the pandemic: risk assessments, engagement, and a plan for rehabilitation and recovery. She applauded the NHS Health and Race Observatory newly created to look at these matters. ${ }^{3}$ All staff must be risk assessed regardless of background, she said, and all need access to proper PPE-no healthcare professionals working in intensive care have died of covid-19, so there is a clear point around personal protection.

Five simple ways frontline workers can maintain their wellbeing $\overline{\text { From Laurie Santos, professor of psychology and head of Silliman College }}$ at Yale University

- 1 Pause and take three slow, conscious breaths-activate the parasympathetic nervous system

- 2 Practise self-compassion. Studies show that devoting five minutes a day to actively thinking "may I be happy, may I (or colleagues) be safe" reduces self-flagellation and reduces burnout

- 3 Feel grateful. Taking a moment to consider what we are appreciative of helps us remember the things we often take for granted. Consider a daily gratitude journal 
- 4 Be more other oriented-research shows that if we put our money and our time into helping other people, it can make us feel happier. So, spend a few pounds helping a local business or take time to reach out to a friend. Actively prioritising your social connections is also important now that we're not naturally seeing people in real life

- 5 Lower your expectations of others-when people don't feel they are walking on eggshells, it gives both you and them the benefit of the doubt

The conference is free to watch at www.riskybusiness.events/lessons-from-covid-19-zoom-conference

1 Wilkinson E. RECOVERY trial: the UK covid-19 study resetting expectations for clinical trials. BMJ 2020;369:m1626. doi: 10.1136/bmj.m1626 pmid: 32345591

2 lacobucci G. Covid-19: review of ethnic disparities is labelled "whitewash" for lack of recommendations. BMJ 2020;369:m2208doi: 10.1136/bmj.m2208.

3 Kmietowicz Z. NHS launches Race and Health Observatory after BM's call to end inequalities. BMJ 2020;369:m2191. doi: 10.1136/bmi.m2191 pmid: 32482618

This article is made freely available for use in accordance with BMJ's website terms and conditions for the duration of the covid-19 pandemic or until otherwise determined by BMI. You may use, download and print the article for any lawful, non-commercial purpose (including text and data mining) provided that all copyright notices and trade marks are retained. 